\title{
Orbital Lymphoproliferative Disorders (OLPDs): Value of MR Imaging for Differentiating Orbital Lymphoma from Benign OPLDs
}

\author{
K. Haradome, H. Haradome, Y. Usui, S. Ueda, T.C. Kwee, K. Saito, K. Tokuuye, J. Matsubayashi, T. Nagao, and H. Goto
}

\begin{abstract}
BACKGROUND AND PURPOSE: Accurate discrimination of orbital lymphoma from benign orbital lymphoproliferative disorders is crucial for treatment planning. We evaluated MR imaging including DWI and contrast-enhanced MR imaging for differentiating orbital lymphoma from benign orbital lymphoproliferative disorders.

MATERIALS AND METHODS: Forty-seven histopathologically proved orbital lymphoproliferative disorders (29 orbital lymphomas and 18 benign orbital lymphoproliferative disorders) were evaluated. Two board-certified radiologists reviewed visual features on T1-weighted, fat-suppressed T2-weighted, diffusion-weighted, and contrast-enhanced MR images. For quantitative evaluation, ADC and contrastenhancement ratio of all lesions were measured and optimal cutoff thresholds and areas under curves for differentiating orbital lymphoma from benign orbital lymphoproliferative disorders were determined using receiver operative characteristic analysis; corresponding sensitivities and specificities were calculated.

RESULTS: Multivariate logistic regression analysis showed that ill-defined tumor margin $(P=.003)$ had a significant association with orbital lymphoma whereas the "flow void sign" $(P=.005)$ and radiologic evidence of sinusitis $(P=.0002)$ were associated with benign orbital lymphoproliferative disorders. The mean ADC and contrast-enhancement ratio of orbital lymphomas were significantly lower than those of benign orbital lymphoproliferative disorders $(P<.01)$. An ADC of less than $0.612 \times 10^{-3} \mathrm{~mm}^{2} / \mathrm{s}$ and a contrast-enhancement ratio of less than 1.88 yielded areas under curves of 0.980 and 0.770 , sensitivity of $94.1 \%$ and $95.5 \%$, and specificities of $93.3 \%$ and $80.0 \%$ for predicting orbital lymphoma, respectively.
\end{abstract}

CONCLUSIONS: Some characteristic MR imaging features and quantitative DWI and contrast-enhanced MR imaging are useful in further improving the accuracy of MR imaging for differentiation of orbital lymphoma from benign orbital lymphoproliferative disorders.

ABBREVIATIONS: AUC = area under curve; CER = contrast-enhancement ratio; ICC = interclass correlation coefficient; MALT = extranodal marginal zone lymphoma of mucosa-associated lymphoid tissue; OLPD = orbital lymphoproliferative disorder

O rbital lymphoproliferative disorders (OLPDs) frequently present as an orbital mass lesion $(24 \%-49 \%)$ in the adult and comprise a wide spectrum of diseases ranging from benign to malignant lesions. ${ }^{1}$ Orbital lymphoma is the most common orbital neoplasm representing $55 \%$ of cases in adults. ${ }^{2}$ Most orbital lymphomas are primary, low-grade, B-cell, non-Hodgkin lymphomas, and the most common subtype is extranodal marginal

Received February 11, 2014; accepted after revision March 22.

From the Departments of Ophthalmology (K.H., Y.U., S.U., H.G.), Radiology (K.S., K.T.), and Anatomic Pathology (J.M., T.N.), Tokyo Medical University, Tokyo, Japan; Department of Radiology (H.H.), Nihon University School of Medicine, Tokyo, Japan; and Department of Radiology (T.C.K.), University Medical Center Utrecht, Utrecht, the Netherlands.

Please address correspondence to Hiroki Haradome MD, PhD, 30-1, Ohyaguchi Kami-cho Itabashi-ku, Tokyo 173-8610, Japan; e-mail: karate.b@gmail.com

- Indicates open access to non-subscribers at www.ajnr.org

http://dx.doi.org/10.3174/ajnr.A3986 zone lymphoma of mucosa-associated lymphoid tissue (MALT). ${ }^{3}$ Other OLPDs comprise several benign, noninfectious, chronic inflammatory diseases, including IgG4-related ophthalmic disease, reactive lymphoid hyperplasia, and idiopathic orbital inflammation. ${ }^{4}$ Among them, IgG4-related ophthalmic disease is becoming increasingly recognized and accounts for approximately half of benign OPLDs on the basis of recent surveillance. ${ }^{5}$ The discrimination of orbital lymphoma from benign OLPDs is crucial because of the different therapeutic implications: the former is amenable to low-dose radiation therapy, whereas the latter are expected to show a good response to corticosteroid therapy. ${ }^{6}$ The utility of conventional anatomic MR imaging for this purpose is limited, however, because orbital lymphoma and benign OLPDs frequently share similar imaging features. ${ }^{7,8}$ Recently, some researchers have reported quantitative DWI with ADC measurements to be potentially useful for discriminating orbital lymphoma from other orbital tumors. ${ }^{9-15}$ However, these studies in- 
cluded other neoplastic and nonneoplastic lesions such as cavernous hemangiomas, neurogenic tumors, and metastases, and the value of DWI for discrimination of lymphoma and OLPDs remains unclear. The purpose of this study was to assess the value of MR imaging including DWI and contrast-enhanced MR imaging for the discrimination of primary orbital lymphoma from benign OLPDs.

\section{MATERIALS AND METHODS}

\section{Patients}

The institutional review board of Tokyo Medical University approved this retrospective study. Written informed consent from patients was waived.

A total of 187 patients with an orbital mass lesion and whom had undergone surgical biopsy for pathologic diagnosis were identified in our hospital's database within a consecutive period of 5 years (between January 2008 and September 2013). From these pathologically-proved cases, we retrospectively searched for eligible candidates for this study using the search terms "lymphoproliferative disorder including malignant lymphoma, IgG4related ophthalmic disease, reactive lymphoid hyperplasia, and idiopathic orbital inflammation," and 77 patients were identified. Of these 77 patients, 30 patients were excluded because of 1 ) nonavailability of both DWI and contrast (gadolinium)-enhanced MR imaging $(n=16), 2)$ administration of corticosteroid therapy before MR imaging $(n=10), 3)$ a case of secondary lymphoma $(n=1), 4)$ a case with inconclusive pathology $(n=1)$, and 5) two cases with a single small lesion at the conjunctiva that did not allow for accurate ADC measurement. Finally, 47 patients (28 men and 19 women; mean age and standard deviation $66.6 \pm 14.4$ years; age range, $27-88$ years) with 47 lesions (29 orbital lymphomas and 18 benign OLPDs) were enrolled in this study. Benign OLPDs consisted of IgG4-related ophthalmic disease $(n=14)$ and reactive lymphoid hyperplasia $(n=4)$. In the cohort, 27 patients had undergone both DWI and contrast-enhanced MR examinations, 5 patients had undergone only DWI, and 15 patients had undergone only contrast-enhanced MR imaging. All patients received the MR imaging before surgical biopsy.

\section{MR Imaging}

All MR examinations were performed using a $1.5 \mathrm{~T}$ scanner (Avanto; Siemens, Erlangen, Germany) equipped with highperformance gradients (maximum gradient strength of $45 \mathrm{mT} / \mathrm{m}$; peak slew rate of $200 \mathrm{mT} / \mathrm{m} / \mathrm{ms}$ ) and a 12-channel phased-array head coil allowing for parallel image acquisition.

Unenhanced MR images included 1) transverse and coronal T2-weighted fat-suppressed turbo spin-echo sequences (TR/TE of $3500 \mathrm{~ms} / 96 \mathrm{~ms}$, echo-train length of 7 , section thickness of 3 $\mathrm{mm}$, an intersection gap of $0.3 \mathrm{~mm}(10 \%)$, a field of view of $150 \times$ $150 \mathrm{~mm}^{2}$, matrix of $320 \times 320$, number of excitations of 2);2) a transverse T1-weighed spin-echo sequence (TR/TE of $450 \mathrm{~ms} / 12$ $\mathrm{ms}$, flip angle of 90 , section thickness of $3 \mathrm{~mm}$, an intersection gap of $0.3 \mathrm{~mm}(10 \%)$, field of view of $150 \times 150 \mathrm{~mm}^{2}$, matrix of $512 \times$ 512 , number of excitations of 2); and 3) a transverse diffusionweighted sequence with b-values of 0 and 1000 seconds $/ \mathrm{mm}^{2}$ (TR/TE of $6836 \mathrm{~ms} / 68 \mathrm{~ms}$, section thickness of $2 \mathrm{~mm}$, an intersection gap of $0.2 \mathrm{~mm}(10 \%)$, a field of view of $280 \times 280 \mathrm{~mm}^{2}$, matrix of $256 \times 208$, number of excitations of $6-8$ ). Parallel imaging acquisition with a reduction factor of 2 was applied for DWI. Diffusion motion probing gradients were applied in 3 orthogonal directions and trace images were obtained using the manufacturer's software. Voxel-based ADC maps were created by means of a commercial workstation on the MR console using a linear regression analysis of the function $S=S_{0} \times \exp (-b \times$ ADC), where $S$ is the signal intensity after the applied motion probing gradients and $S_{0}$ is the signal intensity at $b=0$ seconds/ $\mathrm{mm}^{2}$. Two b-values ( 0 and 1000 seconds $/ \mathrm{mm}^{2}$ ) were used for the ADC calculation. After intravenous administration of an extracellular gadolinium-based contrast agent $(0.2 \mathrm{~mL} / \mathrm{kg})$, transverse, coronal, and sagittal contrast-enhanced fat-saturated T1weighted sequences (TR/TE of $632 \mathrm{~ms} / 12 \mathrm{~ms}$, flip angle of 90, section thickness of $3 \mathrm{~mm}$, intersection gap of $0.3 \mathrm{~mm} \mathrm{[10 \% ],} \mathrm{a}$ field of view of $150 \times 150 \mathrm{~mm}^{2}$, matrix of $512 \times 512$, number of excitations of 2) were obtained.

\section{Qualitative MR Imaging Features Analysis}

All MR images were reviewed by 2 board-certified radiologists with 19 and 9 years of experience, respectively, who were blinded to the clinical information and histologic results, using a PACS. Consensus between the 2 readers was reached by means of an additional joint reading session. The following items were evaluated: 1) the laterality (unilateral/bilateral), 2) the shape of the margins (well-defined, ill-defined [infiltrative], and lobulated) and signal intensity on T1-weighted, T2-weighted, and diffusionweighted images relative to cerebral cortex (hypo-, iso-, or hyperintense), ${ }^{10,11} 3$ ) the homogeneity and degree of contrast enhancement relative to extraocular muscles, 4) the presence of a signal void from a vessel in the lesion on T2-weighted images, referred to as the "flow void sign," and 5) findings indicative of sinusitis; for this purpose, the readers comprehensively considered the following criteria: 1) significant paranasal mucosal thickness ( $>4 \mathrm{~mm})$, 2) fluid level, and 3) the presence of a retention cyst at each paranasal cavity. ${ }^{16}$

\section{Quantitative DWI and Contrast-Enhanced MR Imaging Analyses}

Regions of interest for ADC measurements and contrastenhancement ratio (CER) calculation were determined by an ophthalmologist assisted by a radiologist with 20 years of clinical experience. Both observers were blinded to the pathologic results. On all lesions, a circular region of interest was placed over the entire lesion on "diffusion-weighted" images obtained with a bvalue of 0 seconds $/ \mathrm{mm}^{2}$, while avoiding artifacts and in reference to the other sequences. This region of interest was then copied and pasted onto the ADC maps and the corresponding ADCs were measured. ADC measurements were repeated 3 times for each lesion and the mean was calculated for further statistical analysis. To calculate the CER, the signal intensities of the lesions and temporal muscle were measured for each patient on transverse fat-saturated contrastenhanced T1-weighted images. The signal intensity of the temporal muscle was measured by a circular region of interest while avoiding the artifacts. The CERs were calculated as follows: $S I_{\text {lesion }} / S I_{\text {temporal muscle, }}$, where $S I_{\text {lesion }}$ and $S I_{\text {temporal muscle }}$ are the signal intensity of the lesion and the temporal muscle on contrast-enhanced 
Table 1: Patient characteristics and pathologic findings of OLPD cases

\begin{tabular}{|c|c|c|c|}
\hline & Orbital Lymphoma $(n=29)$ & Benign OLPDs $(n=18)$ & $P$ Value \\
\hline Age & $72.2 \pm 11.3(47-88)$ & $57.6 \pm 14.7(27-80)$ & $P=.001^{\mathrm{a}}$ \\
\hline Sex & Male 18, Female 11 & Male 10, Female 8 & $P=.763$ \\
\hline \multirow[t]{4}{*}{ Histologic subtypes } & MALT lymphoma 21 (72) & $\begin{array}{l}\text { IgG4-related ophthalmic } \\
\text { disease } 14 \text { (78) }\end{array}$ & \\
\hline & DLBCL 4 (14) & RLH 4 (22) & \\
\hline & Follicular lymphoma 3 (10) & & \\
\hline & Mantle cell lymphoma 1 (3) & & \\
\hline \multirow[t]{2}{*}{ Laterality } & Bilateral $12(41)$ & Bilateral 15 (83) & $P=.006^{\mathrm{a}}$ \\
\hline & Unilateral 17 (59) & Unilateral 3 (17) & \\
\hline
\end{tabular}

Note:-Data in parentheses are ranges for age and percentages for histologic subtypes and laterality. DLBCL indicates diffuse large B-cell lymphoma; RLH, reactive lymphoid hyperplasia.

a There were significant differences in age and laterality between the 2 groups.

Table 2: MR imaging characteristics

\begin{tabular}{|c|c|c|c|}
\hline & $\begin{array}{l}\text { Orbital Lymphoma } \\
\qquad(n=29)\end{array}$ & $\begin{array}{l}\text { Benign OLPDs } \\
(n=18)\end{array}$ & $P$ Value \\
\hline Shape & $\begin{array}{l}\text { Well-defined } 7(24) \\
\text { Ill-defined } 21(72) \\
\text { Lobulated } 1(3)\end{array}$ & $\begin{array}{l}\text { Well-defined } 10 \text { (56) } \\
\text { Ill-defined } 3 \text { (17) } \\
\text { Lobulated } 5 \text { (17) }\end{array}$ & $P=.0006^{\mathrm{a}}$ \\
\hline \multicolumn{4}{|l|}{ Signal intensity } \\
\hline TIWI & $\begin{array}{l}\text { Iso } 28 \text { (97) } \\
\text { Low } 1 \text { (3) }\end{array}$ & Iso 18 (100) & $P=.2548$ \\
\hline $\mathrm{T} 2 \mathrm{WI}$ & $\begin{array}{l}\text { Iso } 27(93) \\
\text { Low } 2 \text { (7) }\end{array}$ & $\begin{array}{l}\text { Iso } 16(89) \\
\text { Low } 2 \text { (11) }\end{array}$ & $P=.6147$ \\
\hline DWI & High 17 (100) & $\begin{array}{l}\text { High } 14(93) \\
\text { Iso } 1(7)\end{array}$ & $P=.2794$ \\
\hline \multirow[t]{2}{*}{$\begin{array}{l}\text { Homogeneity and degree of } \\
\text { contrast enhancement }\end{array}$} & $\begin{array}{l}\text { Homogeneous } 23 \text { (85) } \\
\text { Inhomogeneous } 4 \text { (15) }\end{array}$ & Homogeneous 15 (100) & $P=.2787$ \\
\hline & Iso $27(100)$ & $\begin{array}{l}\text { High } 2(13) \\
\text { Iso } 13(87)\end{array}$ & $P=.0519$ \\
\hline Presence of "flow void sign" & $17(59)$ & $17(94)$ & $P=.0084^{\mathrm{a}}$ \\
\hline Findings suggestive of sinusitis & $8(28)$ & $16(89)$ & $P<.001^{\mathrm{a}}$ \\
\hline
\end{tabular}

MR images, respectively. ${ }^{17}$ To confirm the reproducibility of both $\mathrm{ADC}$ and CRE measurements for the intraobserver agreement analysis, 3 repeated measurements were conducted with a week interval between each set of measurements.

\section{Pathologic Diagnosis}

Pathologic diagnosis of all OLPDs was established using histopathologic features including immunohistochemical staining, flow cytometric analysis, and gene rearrangement analysis according to the latest World Health Organization ${ }^{18}$ criteria in 2008. Flow cytometric and gene rearrangement analyses were used to identify a monoclonal immunoglobulin band for the diagnosis of orbital lymphoma. For the diagnosis of IgG4-related ophthalmic disease, the following 2 main criteria were adopted: 1 ) serum IgG4 concentration $>135 \mathrm{mg} / \mathrm{dl}$, and 2) $>40 \%$ of IgG4 positive plasma cells and $>10$ cells/high-power field in the biopsy sample. ${ }^{18}$ The distributions of B- and T-cells in a lesion were evaluated by immunohistochemical staining, where a normal distribution of these cells was considered to indicate reactive lymphoid hyperplasia.

\section{Statistical Analysis}

All OLPDs were divided into 2 groups: orbital lymphoma and benign OLPDs. The differences in mean age and sex distinction between the 2 groups were assessed by a $t$-test and a Fisher exact test, respectively. The results of the qualitative MR features between the 2 groups were compared using a $\chi^{2}$ test. The parameters found to have statistical significance by univariate analysis were entered into a multivariate logistic regression model to elucidate the useful findings for predicting orbital lymphoma or benign OLPDs. Differences in mean lesion ADCs and CERs between the 2 groups were assessed using a $t$-test with a Welch correction. Receiver operating characteristic analysis with multiple logistic regression was used to calculate areas under the ROC curve (AUC) and optimal cutoff thresholds of ADC and CER for differentiating orbital lymphoma from benign OLPDs, along with corresponding sensitivities, specificities, and positive and negative predictive values. The intrarater reliability was calculated by an interclass correlation coefficient (ICC) using a random effect analysis of variance. Agreement between the 2 readers with regard to the assessment of qualitative MR features was analyzed using the $\kappa$ statistic. Differences in mean region of interest size in ADC and CER measurements between the 2 groups were analyzed using the Mann-Whitney test. The $P$ values $<.05$ were considered to indicate a significant difference.

\section{RESULTS}

\section{Patient Characteristics, Pathologic Findings, Disease Distribution}

Patient characteristics, pathologic subtypes, and disease distribution are summarized in Table 1. Age of the patients with orbital lymphoma was significantly higher than those with benign OLPDs ( $P=$ $.001)$ whereas no significant difference in sex distribution was observed between the 2 groups $(P=.763)$. Most orbital lymphomas and benign OLPDs were MALT lymphoma (21/29, 72\%) and IgG4related ophthalmic disease $(14 / 18,78 \%)$, respectively. Most orbital lymphomas had unilateral involvement (17/29,59\%), whereas most benign OLPDs had bilateral involvement (15/18, 83\%).

\section{Qualitative MR Imaging Features}

The qualitative MR imaging features of orbital lymphoma and benign OLPDs are summarized in Table 2. Most orbital lymphomas and benign OLPDs appeared as ill-defined masses (21/29, $72 \%)$ and well-defined masses $(10 / 18,56 \%)$, respectively, and a significant difference was observed between the 2 groups $(P=$ .0006). The lesion signal of orbital lymphoma and benign OLPDs on unenhanced MR imaging was similar, and most lesions showed isointensity on T1-weighted and T2-weighted images and 

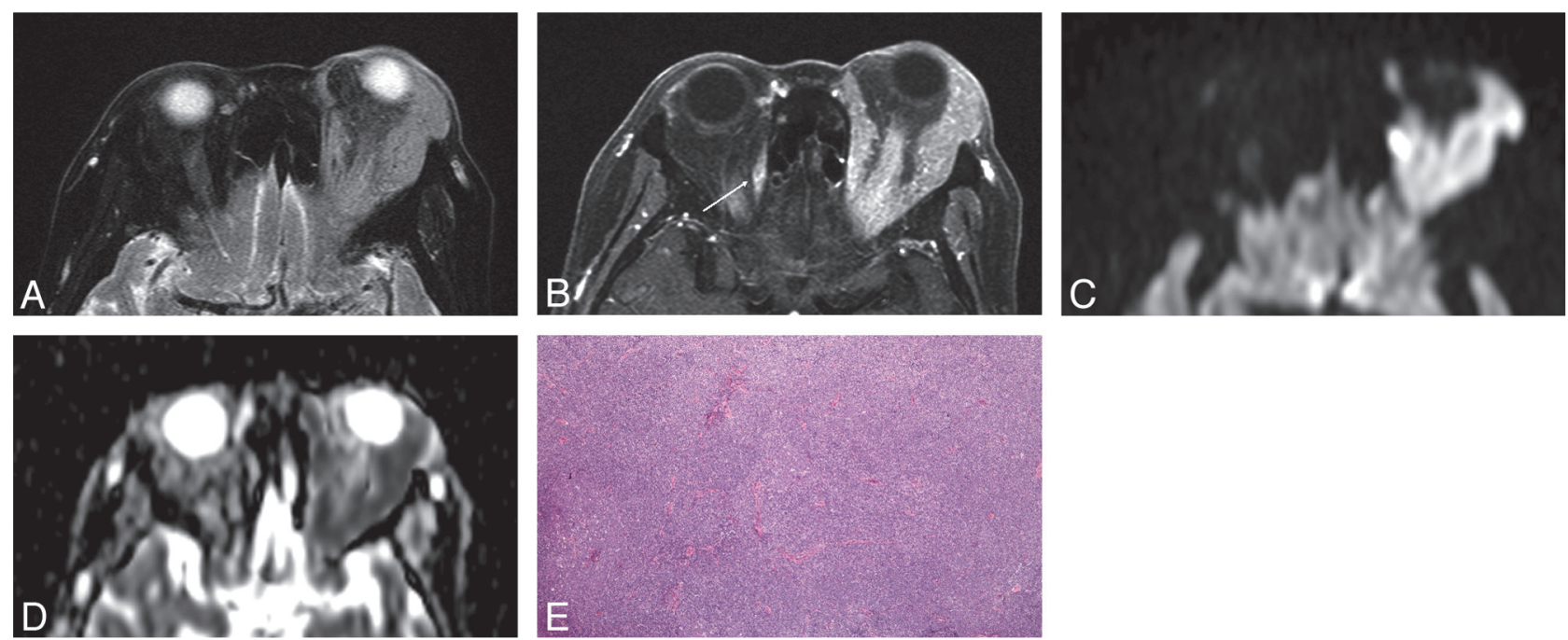

FIG 1. Orbital lymphoma (MALT lymphoma) in a 79-year-old woman. $A$, Transverse fat-saturated T2-weighted image; $B$, Transverse postcontrast fat-saturated T1-weighted image; $C$, Transverse DWI; D, ADC map; and E, Hematoxylin and eosin (HE)-stained biopsied specimen of the orbital tumor at low power field. An infiltrative mass involves the left lacrimal gland and extraocular muscles, appears isointense compared with the brain cortex on a fat-saturated T2-weighted MR image $(A)$, and shows homogeneous contrast enhancement, similar to that of the right extraocular muscle (arrow) (B). The mass appears as strongly hyperintense on DWI $(C)$ and hypointense on the ADC map (D). Notably, noninvolved extraocular muscles do not show hyperintensity similar to that of the mass on DWI (C). Lesion ADC and CER were $0.56 \times 10^{-3}$ $\mathrm{mm}^{2} / \mathrm{s}$ and 1.69 , respectively. HE-stained biopsied specimen $(E)$ demonstrated numerous small-to-medium-sized atypical lymphocytes around reactive lymph follicles, with greater high cellular attenuation, however, interstitial fibrosis or edematous change was not prominent.
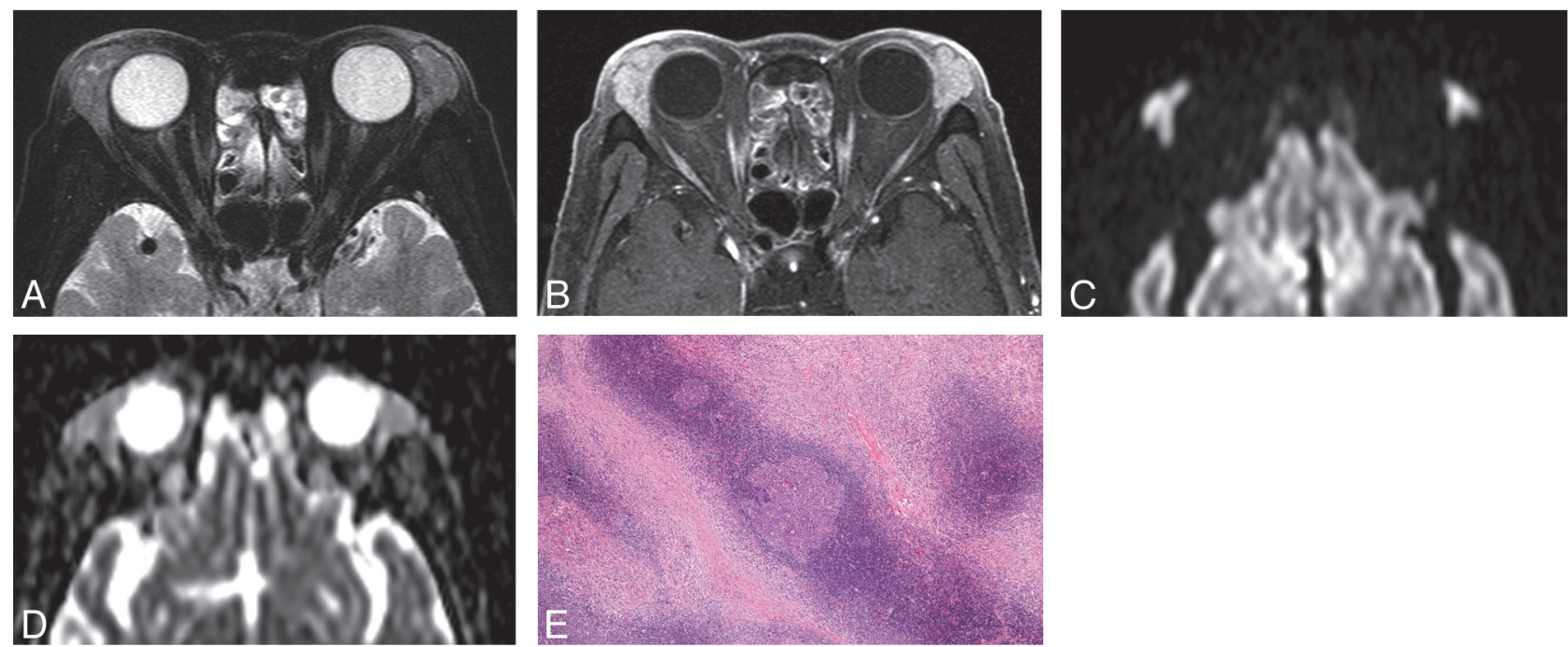

FIG 2. IgG4-related ophthalmic disease in a 67-year-old woman. $A$, Transverse fat-saturated T2-weighted image; $B$, transverse postcontrast fat-saturated T1-weighted image; $C$, transverse DWI; $D, A D C$ map; and $E$, Hematoxylin and eosin (HE)-stained biopsied specimen of the lacrimal gland at low power field. The mass-like enlarged lacrimal glands are isointense compared with the brain cortex on the fat-saturated T2-weighted image $(A)$ and show homogeneous contrast enhancement, similar to that of the extraocular muscles (B). The lesions appear mildly hyperintense

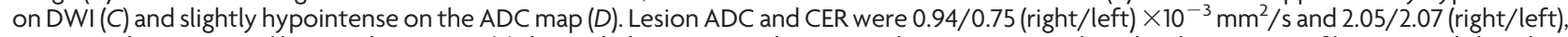
respectively. HE-stained biopsied specimen $(E)$ showed a large germinal center with accompanying lymphoplasmacytic infiltration and abundant interstitial fibrosis with edematous changes. On immunochemical stained section analysis (not shown), many lgG4-positive plasma cell (>40\%) were identified, and this is compatible with IgG4-related ophthalmic disease.

hyperintensity on diffusion-weighted images, relative to cerebral cortex (Figs 1-3). In the 2 benign OLPDs showing hypointensity on T2-weighted images, abundant fibrotic components (sclerosed type) were histopathologically identified. After gadoliniumbased contrast administration, most orbital lymphomas and all benign OLPD lesions showed homogeneous contrast enhancement $(23 / 27,85 \%$ for orbital lymphomas and 15/15, 100\% for benign OLPDs) and their signal intensities were similar to those of the nonaffected external ocular muscles (Figs 1-3). The "flow void sign" (Fig 4) was identified significantly more frequently $(P=.008)$ in benign OLPDs $(17 / 18,94 \%)$ than in orbital lymphoma $(17 / 29,59 \%)$. Radiologic sinusitis findings were also seen significantly more often $(P<.001)$ in benign OLPDs $(16 / 18$, $89 \%)$ than in orbital lymphoma $(8 / 29,28 \%)$. Upon multivariate logistic regression analysis, ill-defined tumor margin $(P=.003)$ was a significant predictor of orbital lymphoma, whereas the "flow void sign" $(P=.005)$ and radiologic sinusitis $(P=.0002)$ were predictors of benign OLPDs. The reader agreement for the 

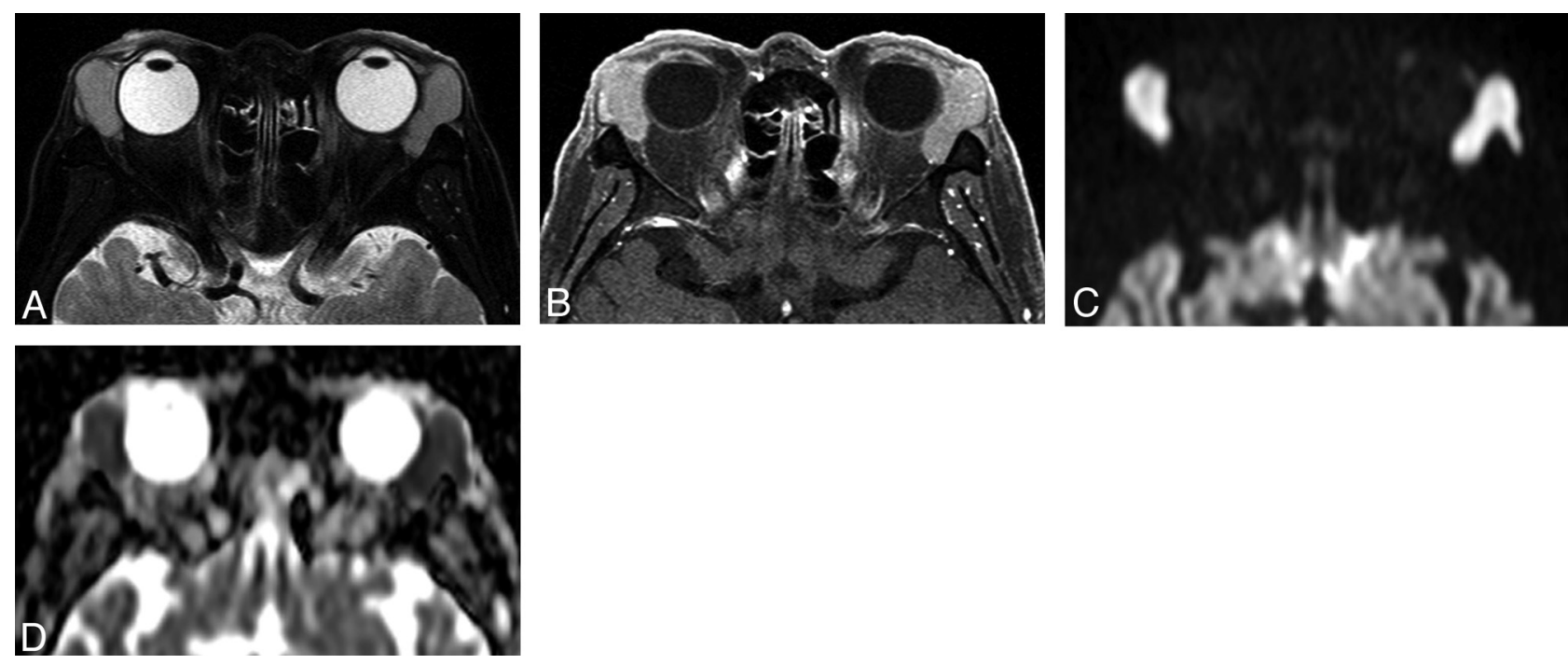

FIG 3. Orbital lymphoma (mantle cell lymphoma) in a 71-year-old man. $A$, Transverse fat-saturated T2-weighted image; $B$, transverse fatsaturated postcontrast T1-weighted image; $C$, transverse DWI; and $D, A D C$ map. The lesions involve the bilateral lacrimal glands, appear isointense compared with the brain cortex on fat-saturated T2-weighted image (A), and show homogeneous contrast enhancement, similar that of the extraocular muscles $(B)$. The lesions are strongly hyperintense on the DWI $(C)$ and hypointense on the ADC map (D). Lesion ADC and CER were $0.47 / 0.48$ (right/left) $\times 10^{-3} \mathrm{~mm}^{2} / \mathrm{s}$ and 1.49/1.46 (right/left), respectively. The low ADC and CER values could suggest orbital lymphoma though the imaging features are similar to that of IgG4-related ophthalmic disease (Fig 2).

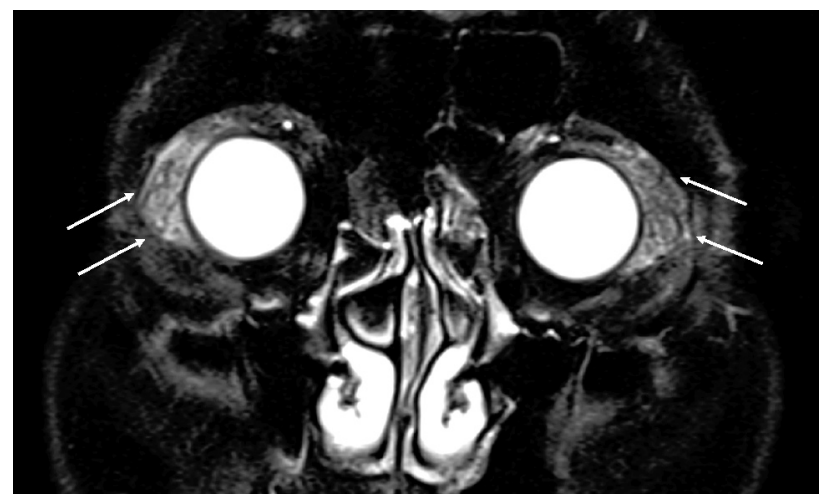

FIG 4. Reactive lymphoid hyperplasia in a 65-year-old man. $A$, Coronal fat-saturated T2-weighted image. A number of vessel signal voids are observed in the bilaterally enlarged lacrimal glands on T2weighted image ("flow void sign") (arrows).

assessment of qualitative MR imaging features was good to excellent (0.627-1.00): shape, 0.856; T1WI signal, 0.849; T2WI signal, 0.877; DWI signal, 1.00; degree of contrast enhancement, 0.916; homogeneity of contrast enhancement, 0.627; flow void sign, 0.787 ; radiologic sinusitis, 0.872 . Additional joint reading sessions had to be conducted to obtain the consensus in up to a maximum of 4 cases for each qualitative MR imaging feature.

\section{Quantitative DWI and Contrast-Enhanced MR Imaging Analyses}

The ADCs and CERs of orbital lymphoma and benign OLPDs, including their histologic subtypes, are summarized in Tables 3 and 4. The mean ADC and CER of orbital lymphoma $\left(0.544 \times 10^{-3} \mathrm{~mm}^{2} / \mathrm{s}\right.$ and 1.70$)$ were significantly lower $(P<.01)$ than those of benign OLPDs $\left(0.812 \times 10^{-3} \mathrm{~mm}^{2} / \mathrm{s}\right.$ and 2.07) (Fig 5). The standard deviation of the ADC of orbital lymphomas was lower $( \pm 0.051)$ compared with that of benign OLPDs $( \pm 0.246)$, and ADCs of only 2 benign OLPDs overlapped with those of orbital lymphomas in the
$\mathrm{ADC}$ range, whereas CERs of both entities showed considerable overlap though a significant difference was observed in their mean CERs (Fig 5). Based upon the ROC analysis, an optimal ADC threshold of less than $0.612 \times 10^{-3} \mathrm{~mm}^{2} / \mathrm{s}$ yielded an AUC, sensitivity, and specificity of $0.980,94.1 \%$, and $93.3 \%$, and a positive and negative predictive value of $94.1 \%$ and $93.3 \%$ for predicting orbital lymphoma. Meanwhile, an optimal CER threshold of less than 1.88 yielded an AUC, sensitivity, and specificity of $0.770,95.5 \%$, and $80.0 \%$, and a positive and negative predictive value of $87.5 \%$ and $92.3 \%$ for diagnosing orbital lymphoma. In benign OLPDs, a lower ADC and CER below each optimal threshold (false-positive lesion) were observed with 1 lesion in the ADC and 3 lesions in the CER, respectively. The 1 benign OLPD lesion showing a lower ADC demonstrated pathologically a relatively higher cellular attenuation, less edematous change, and a mild to moderate fibrosis. In the 3 benign OLPD lesions showing a lower CER, fibrotic changes were less prominent than in the other benign OLPDs, whereas the orbital lymphomas showing a higher CER $(>2.0)$ had a tendency to have higher vascularization on pathology. Intraobserver reliabilities were almost perfect in the repeated ADC and CER measurements: ADC in orbital lymphoma (ICC $=0.98, P<.001$ ), ADC in benign OLPDs (ICC $=$ $0.99, P<.001)$, CER in orbital lymphoma $(\mathrm{ICC}=0.97, P<.001)$, and CER in benign OLPDs (ICC $=0.98, P<.001$ ). The region of interest sizes for $\mathrm{ADC}$ and CER measurements ranged from 34.6 to $221.4 \mathrm{~mm}^{2}$ (mean, $69.6 \mathrm{~mm}^{2}$ ) and from 22.1 to $268.2 \mathrm{~mm}^{2}$ (mean, $85.2 \mathrm{~mm}^{2}$ ), respectively. The mean region of interest sizes for both $\mathrm{ADC}$ and CER measurements in benign OLPDs $\left(59.3 \mathrm{~mm}^{2}\right.$ in ADC, $61.6 \mathrm{~mm}^{2}$ in CER) were smaller than those in orbital lymphomas (78.7 $\mathrm{mm}^{2}$ in ADC, $98.3 \mathrm{~mm}^{2}$ in CER) though no significant differences were observed.

\section{DISCUSSION}

In agreement with previous results, ${ }^{10,11,14}$ we found that the mean ADC of orbital lymphomas $\left(0.544 \times 10^{-3} \mathrm{~mm}^{2} / \mathrm{s}\right)$ was signifi- 
cantly lower $(P<.001)$ than that of benign OLPDs $\left(0.812 \times 10^{-3}\right.$ $\mathrm{mm}^{2} / \mathrm{s}$ ). It is thought to reflect a higher cellularity in orbital lymphoma lesions because of numerous uniformly small-sized atypical lymphocyte infiltrations. ${ }^{19,20}$ Moreover, interstitial edematous changes in benign OLPDs, which lead to increased ADC, could also contribute to a significant difference in the ADCs. It is important to note that previous studies mainly included other benign and malignant orbital neoplastic lesions rather than inflammatory orbital lesions. ${ }^{9-15}$ Sepahdari et al ${ }^{10}$ reported that an ADC of less than $1.0 \times 10^{-3} \mathrm{~mm}^{2} / \mathrm{s}$ was optimal for predicting malignancy and Politi et al ${ }^{11}$ indicated that a threshold of $0.775 \times$ $10^{-3} \mathrm{~mm}^{2} / \mathrm{s}$ was optimal for predicting orbital lymphoma. Therefore, their reported optimal ADC may be unfit for differentiating orbital lymphoma from benign OLPDs because these ADC values

Table 3: ADCs of each group of OLPDs

\begin{tabular}{lccc}
\hline & Mean ADC \pm SD & ADC range & P Value \\
\hline Orbital lymphoma $(n=17)$ & $0.54 \pm 0.05$ & $0.44-0.64$ & $P<.001^{\mathrm{a}}$ \\
MALT lymphoma $(n=12)$ & & $0.47-0.64$ & \\
DLBCL $(n=2)$ & & $0.44-0.56$ & \\
Follicular lymphoma $(n=2)$ & & $0.53-0.57$ & \\
Mantle cell lymphoma $(n=1)$ & 0.47 & \\
Benign OLPDs $(n=15)$ & $0.81 \pm 0.18$ & $0.58-1.24$ & \\
IgG4-related ophthalmic disease $(n=12)$ & & $0.58-1.24$ & \\
RLH $(n=3)$ & & $0.73-0.85$ & \\
\hline
\end{tabular}

Note:-There were significant differences in ADC between the 2 groups. SD indicates standard deviation; DLBCL diffuse large B-cell lymphoma; RLH, reactive lymphoid hyperplasia.

Table 4: CERs of each group of OLPDs

\begin{tabular}{lccc}
\hline & Mean CER \pm SD & CER Range & $P$ Value \\
\hline Orbital lymphoma $(n=27)$ & $1.70 \pm 0.25$ & $1.27-2.24$ & $P=.0096^{\mathrm{a}}$ \\
MALT lymphoma $(n=20)$ & & $1.27-2.24$ & \\
DLBCL $(n=3)$ & & $1.36-1.71$ & \\
Follicular lymphoma $(n=3)$ & & $1.76-1.94$ & \\
Mantle cell lymphoma $(n=1)$ & $2.07 \pm 0.46$ & 1.55 & \\
Benign OLPDs $(n=15)$ & & $1.27-3.03$ & \\
IgG4-related ophthalmic disease $(n=11)$ & & $1.66-2.14$ & \\
RLH $(n=4)$ & & & \\
\hline
\end{tabular}

${ }^{a}$ There were significant differences in CER between the 2 groups. SD indicates standard deviation; DLBCL, diffuse large B-cell lymphoma; RLH, reactive lymphoid hyperplasia.
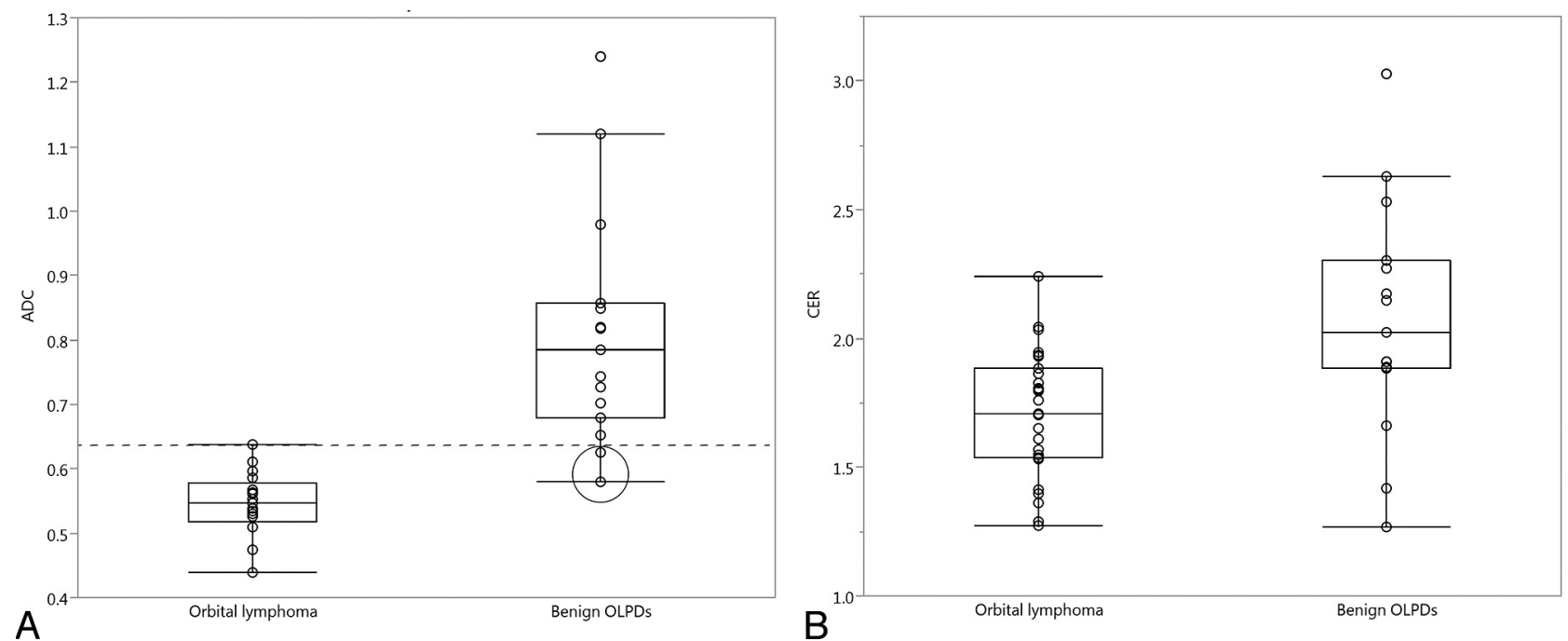

FIG 5. ADCs and CERs of orbital lymphoma and benign OPLDs. $A$ and $B$, Scatterplot and box-and-whisker plot show the distribution of ADCs and CERs, and each mean value in the orbital lymphoma and benign OLPDs lesions. The mean ADC and CER of orbital lymphomas are significantly lower than those of benign OLPDs $(P<.01)$. The standard deviation of the ADC of orbital lymphomas was lower $( \pm 0.051)$ compared with that of benign OLPDs ( \pm 0.246 ), and ADCs of only 2 benign OLPDs (encircled) overlapped with those of orbital lymphomas in the ADC range. measurement error because of contamination from the nonlesional signal in the scanning section. Moreover, orbital lesions are sometimes of small size. For example, if a lesion is localized in the lacrimal gland, it is usually smaller than other orbital lesions. Thus, scanning with thinner section thickness is crucial to reduce the effect of the partial-volume effect on the ADC measurements. In the present study, we used a high b-value (1000 seconds $/ \mathrm{mm}^{2}$ ) and a thinner section thickness (2 mm) for DWI to minimize perfusion and partial volume efglobally lower than those found in previous investigations. In the present study, orbital lymphomas had highly homogeneous ADCs with a low standard deviation, which is in line with previously reported data, ${ }^{11}$ and ADCs of only 2 benign OLPD lesions had values fects. Thus, our ADC values were 
that overlapped with the orbital lymphoma lesions. The mean CER of orbital lymphoma (1.70) was significantly lower $(P=$ .0096) than that of benign OLPDs (2.07), which could be used as an additional valuable index for the diagnosis of orbital lymphoma. Abundant fibrotic components and higher vascularization in benign OLPDs compared with orbital lymphoma may be responsible for the higher CER of benign OLPDs. However, CERs of both entities showed considerable overlap though a significant difference was observed in the mean CER between the 2 entities. We also confirmed the reproducibility of the measured $\mathrm{ADC}$ and CER values with almost perfect ICC as previously reported. ${ }^{21}$ Ill-defined tumor margin had a significant association with orbital lymphoma in present study. In addition, some characteristic conventional MR imaging features were found to be potentially useful for the differential diagnosis of OLPDs in the present study. The presence of a vessel signal void on T2-weighted MR imaging ("flow void sign") (Fig 4) was observed significantly more frequently $(P=.008)$ in benign OLPDs $(17 / 18$, $94 \%)$ than in orbital lymphoma $(17 / 29,59 \%)$, probably because of the hypervascular nature of the former. ${ }^{22,23}$ As 1 report indicated, an extension of inflammatory changes to the mucosa of nasal and paranasal cavities can occur in IgG4-related disease; ${ }^{24}$ we also found that imaging findings indicative of sinusitis were seen significantly more frequently $(P<.001)$ in benign OLPDs $(16 / 18,89 \%)$ than in orbital lymphoma $(8 / 29,28 \%)$. In addition to the intrinsic limitation of a retrospective study, this study had other limitations that should be noted. First, the number of included patients was relatively low. Further large-scale studies are needed to confirm the present results. Second, in addition to the confirmation of intraobserver reliability with regard to the ADC and CER measurement, assessing interobserver agreement of radiologist versus ophthalmologist would be of interest. Third, further optimization of b-values for DWI in this application may be needed. One researcher adopted a low b-value of 500 seconds $/ \mathrm{mm}^{2} .{ }^{25}$ Although low high b-values may provide higher signal-to-noise ratio and reduce eddy current artifacts, the inclusion of perfusion effects in $\mathrm{ADC}$ measurements could limit their value for discriminating orbital lymphoma from IgG4-related ophthalmic disease because the measured ADCs of both entities showed considerable overlap because of the variation of the lesion vascularity. ${ }^{25}$ Therefore, we believe that the use of a high b-value of 1000 seconds/ $\mathrm{mm}^{2}$ is crucial for accurately distinguishing orbital lymphoma from benign OLPDs with DWI.

\section{CONCLUSIONS}

Some characteristic conventional MR imaging features and quantitative evaluations with DWI and contrast-enhanced MR imaging are useful for discriminating orbital lymphoma from benign OLPDs.

\section{ACKNOWLEDGMENTS}

We are deeply grateful to Takashi Honda, MD (Department of Radiology, Kyorin University School of Medicine), Takashi Shizukuishi, MD (Department of Radiology, Nihon University School of Medicine), and Kazuyoshi Sasakii, RT (Department of Radiology, Tokyo Medical University) for various support on this research.

\section{REFERENCES}

1. Goto H. Review of Ocular Tumor in Practical Ophthalmology. Vol. 24. Tokyo, Japan: Bunkodo; 2008:5-7

2. Valvassori GE, Sabnis SS, Mafee RF, et al. Imaging of orbital lymphoproliferative disorders. Radiol Clin North Am 1999;37:135-50
3. Jenkins C, Rose G, Bunce C, et al. Histological features of ocular adnexal lymphoma (REAL classification) and their association with patient morbidity and survival. Br J Ophthalmol 2000;84:907-13

4. Espinoza GM. Orbital inflammatory pseudotumors: etiology, differential diagnosis, and management. Curr Rheumatol Rep 2010;12:443-47

5. Takahira M, Ozawa Y, Kawano M, et al. Clinical aspects of IgG4related orbital inflammation in a case series of ocular adnexal lymphoproliferative disorders. Int J Rheumatol 2012;2012:635473

6. Garner A. Orbital lymphoproliferative disorders. Br J Ophthalmol 1992;76:47-48

7. Priego G, Majos C, Climent F, et al. Orbital lymphoma: imaging features and differential diagnosis. Insights Imaging 2012;3:337-44

8. Akansel G, Hendrix L, Erickson BA, et al. MRI patterns in orbital malignant lymphoma and atypical lymphocytic infiltrates. Eur J Radiol 2005;53:175-81

9. Kapur R, Sepahdari AR, Mafee MF, et al. MR imaging of orbital inflammatory syndrome, orbital cellulitis, and orbital lymphoid lesions: the role of diffusion-weighted imaging. AJNR Am J Neuroradiol 2009;30:64-70

10. Sepahdari AR, Aakalu VK, Setabutr P, et al. Indeterminate orbital masses: restricted diffusion at MR imaging with echo-planar diffusion-weighted imaging predicts malignancy. Radiology 2010;256:554-64

11. Politi LS, Forghani R, Godi C, et al. Ocular adnexal lymphoma: diffusion-weighted MR imaging for differential diagnosis and therapeutic monitoring. Radiology 2010;256:565-74

12. Roshdy N, Shahin M, Kishk H, et al. MRI in diagnosis of orbital masses. Curr Eye Res 2010;35:986-91

13. Razek AA, Elkhamary S, Mousa A. Differentiation between benign and malignant orbital tumors at 3-T diffusion MR-imaging. Neuroradiology 2011;53:517-22

14. Sepahdari AR, Kapur R, Aakalu VK, et al. Diffusion-weighted imaging of malignant ocular masses: initial results and directions for further study. AJNR Am J Neuroradiol 2012;33:314-19

15. Sepahdari AR, Politi LS, Aakalu VK, et al. Diffusion-weighted imaging of orbital masses: multi-institutional data support a 2-ADC threshold model to categorize lesions as benign, malignant, or indeterminate. AJNR Am J Neuroradiol 2014;35:170-75

16. Bhattacharyya N. The role of CT and MRI in the diagnosis of chronic rhinosinusitis. Curr Allergy Asthma Rep 2010;10:171-74

17. Lee KC, Moon WK, Chung JW, et al. Assessment of lymph node metastases by contrast-enhanced MR imaging in a head and neck cancer model. Korean J Radiol 2007;8:9-14

18. World Health Organization. Classification of Tumors of Heamatopoietic and Lymphoid Tissues. Lyon, France: International Agency for Research on Cancer; 2008

19. Guo AC, Cummings TJ, Dash RC, et al. Lymphomas and high-grade astrocytomas: comparison of water diffusibility and histologic characteristics. Radiology 2002;224:177-83

20. Wu X, Pertovaara H, Dastidar P, et al. ADC measurements in diffuse large B-cell lymphoma and follicular lymphoma: a DWI and cellularity study. Eur J Radiol 2013;82:e158-64

21. Kwee TC, Takahara T, Luijten PR, et al. ADC measurements of lymph nodes: inter- and intra-observer reproducibility study and an overview of the literature. Eur J Radiol 2010;75:215-20

22. Go H, Kim JE, Kim YA, et al. Ocular adnexal IgG4-related disease: comparative analysis with mucosa-associated lymphoid tissue lymphoma and other chronic inflammatory conditions. Histopathology 2012;60:296-312

23. Asai S, Okami K, Nakamura N, et al. Sonographic appearance of the submandibular glands in patients with immunoglobulin G4-related disease. J Ultrasound Med 2012;31:489-93

24. Moteki H, Yasuo M, Hamano $\mathrm{H}$, et al. IgG4-related chronic rhinosinusitis: a new clinical entity of nasal disease. Acta Otolaryngol 2011;131:518-26

25. Hiwatashi A, Yoshiura T, Togao O, et al. Diffusivity of intraorbital lymphoma vs. IgG4-related disease: 3D turbo field echo with diffusion-sensitised driven-equilibrium preparation technique. Eur Radiol 2014;24:581-86 